\title{
COENZYME AND COFACTOR METABOLISM BELONGS TO BIOCHEMICAL PROCESSES SIGNIFICANTLY REGULATED IN HUMAN GRANULOSA CELLS COLLECTED AFTER IVF DURING LONG-TERM PRIMARY IN VITRO CULTURE
}

Mariusz J. Nawrocki ${ }^{1}$, Rafał Sibiak ${ }^{2}$, Maciej Brązert ${ }^{3}$, Piotr Celichowski ${ }^{4}$, Leszek Pawelczyk ${ }^{3}$, Błażej Chermuła $^{3}$, Claudia Dompe ${ }^{5}$, Bartosz Kempisty ${ }^{1,4,6}$, Paul Mozdziak ${ }^{7}$

\begin{abstract}
Granulosa cells (GCs) provide the microenvironment necessary for the development of the follicle and the maturation of the oocyte. GCs are associated with reproductive system function and the maintenance of pregnancy by participating in the synthesis of steroid hormones. Many authors point to new ways of using GCs in regenerative medicine and indicate the significant plasticity of this cell population, suggesting that GCs can undergo a transdifferentiation process. Employing primary in vitro cell cultures and high-throughput transcriptome analysis via Affymetrix microarrays, this study describes groups of genes associated with enzymatic reactions. 52 genes were identified belonging to four gene ontology biological process terms (GO BP): "coenzyme biosynthetic process", "coenzyme metabolic process", "cofactor biosynthetic process" and "cofactor metabolic process". All identified genes showed reduction in the level of mRNA expression during long-term in vitro cultivation. Significanthe transcriptomic profile variability was exhibited for the genes (ELOVL5, ELOVL6 and GPAM) involved in enzymatic regulation of fatty acid metabolism.
\end{abstract}

Running title: Enzymatic regulation in granulosa cells

Keywords: granulosa cells, in vitro culture, coenzyme, cofactor

\footnotetext{
${ }^{1}$ Department of Anatomy, Poznan University of Medical Sciences, Poznan, Poland

2Division of Reproduction, Department of Obstetrics, Gynecology and Gynecologic Oncology, Poznan University of Medical Sciences, Poznan, Poland

${ }^{3}$ Department of Infertility and Reproductive Endocrinology, Poznan University of Medical Sciences, Poznan, Poland

${ }^{4}$ Department of Histology and Embryology, Poznan University of Medical Sciences, Poznan, Poland

${ }^{5}$ The School of Medicine, Medical Sciences and Nutrition, University of Aberdeen, UK

${ }^{6}$ Department of Obstetrics and Gynecology, University Hospital and Masaryk University, Brno, Czech Republic

${ }^{7}$ Physiology Graduate Program, North Carolina State University, Raleigh, North Carolina

* Correspondence: bkempisty@ump.edu.pl

Full list of author information is available at the end of article
} 


\section{Introduction}

Ovarian follicles are formed during the process of folliculogenesis and oogenesis. The follicle consists of the oocyte, the follicular fluid and the oocyte-associated cells: granulosa cells (GCs). GCs provide the microenvironment necessary for the development of the follicle and the maturation of the oocyte [1]. GCs also influence the development of the female gamete in a paracrine and autocrine manner [2]. Paracrine communication leads to the establishment of bidirectional cooperation affecting the hormonal production and the expression of genes associated with follicular development [3]. GCs are associated with the functioning of the reproductive system and the maintenance of pregnancy by participating in the synthesis of steroid hormones. The physiological and morphological changes occurring in GCs during the formation of the ovarian follicle have been well characterized. In contrasts, the deep molecular mechanisms and the gene interactions of GCs are still insufficiently described.

With a large probability for application as the grafts in regenerative medicine, the establishment and characterization of in vitro cell cultures (IVC) may be the most prospective methods of tissue and organ regeneration,. Some studies point to future potential of GCs in regenerative medicine and in transplantology. GCs containing follicular fluid after being transferred for utilization during routine in vitro fertilization procedures may be the source of stem cells. Several authors clearly indicate these previously unidentified properties of GCs. Oppositely, many articles suggest that GCs are subject to a transdifferentiation process [6,7]. During this process, under the influence of the appropriate factors, the mature cell is reprogrammed and it changes into another cell type [4,5]. Kossowska-Tomaszczuk et al. $[6,7]$ indicated that GCs have stem-like properties.

The aim of study is to furtherly characterize GCs at the molecular level and obtain more insight in the variability of the transcriptomic profile during longterm cell culture. Affymetrix microarray assays revealed the regulatory peptides and enzymes involved in the functioning of the mechanism of enzymatic reactions. The focus of the current study was to examine transcript variability of genes belonging to "coenzyme biosynthetic process", "coenzyme metabolic process", "cofactor biosynthetic process" and "cofactor metabolic process" gene ontology biological process terms (GO BP) during OECs longterm primary culture in vitro. It will delineate the transcriptomic profile variability for the genes (ELOVL5, ELOVL6 and GPAM) involved in enzymatic regulation of fatty acid metabolism.

\section{Materials and methods Animals}

A total of 40 crossbred Landrace gilts with a median age of 170 days and weight of $98 \mathrm{~kg}$ were used in this study. All animals were housed under identical conditions.

\section{Collection of porcine ovaries and in vitro culture of granulosa cells}

The ovaries $(n=80)$ and the reproductive tracts were recovered at slaughter and transported to the laboratory at $38^{\circ} \mathrm{C}$ in $0.9 \% \mathrm{NaCl}$ within $30 \mathrm{~min}$. The ovaries of each animal were placed in phosphate-buffered saline supplemented with fetal bovine serum (Sigma-Aldrich Co., St. Louis, MO). Thereafter, single preovulatory large follicles with an estimated diameter $>5 \mathrm{~mm}(\mathrm{n}=300)$ were opened into a sterile Petri dish by puncturing them with a $5 \mathrm{~mL}$ syringe and $20-\mathrm{G}$ needle, recovering the cumulus-oocyte complexes (COCs) and follicular fluid (FF). The GCs were extracted from the FF, whereas the COCs were discarded. Culture medium consisted of Dulbecco's modified Eagle's medium (Sigma-Aldrich) supplemented with $2 \%$ fetal calf serum (PAA, Linz, Austria) and l-glutamine. The final concentration of l-glutamine in the medium was $4 \mathrm{mM}$ (diluted from the $200 \mathrm{mM}$ stock solution; Invitrogen). Moreover, $10 \mathrm{mg} / \mathrm{mL}$ gentamicin (Invitrogen), 10,000 U/mL penicillin, and 10,000 $\mathrm{mg} / \mathrm{mL}$ streptomycin (Invitrogen) were added to the culture medium. Cells were cultivated at $38.5^{\circ} \mathrm{C}$ under aerobic conditions $\left(5 \% \mathrm{CO}_{2}\right)$. Once the adherent cells were $>80 \%$ confluent, they were detached by treating them with $0.05 \%$ trypsin- ethylenediaminetetraacetic acid (Invitrogen) for $3 \mathrm{~min}$ and they were then counted using a Z2 counter and cell viability analyzer (ViCell XR 2.03; both Beckman Coulter). For our experiments, $310^{6}$ cells per dish were used for culture.

\section{RNA extraction from granulosa cells}

Oviductal epithelial cells were pooled and harvested at $24 \mathrm{~h}, 7$ days, 15 days and 30 days after the beginning of the in vitro culture. The total RNA was extracted from samples using TRI Reagent (Sigma, St Louis, MO, USA) according to the method described by Chomczyński and Sacchi [8]. Then the RNA was quantified by measuring the optical density (OD) at $260 \mathrm{~nm}$ (NanoDrop spectrophotometer; Thermo Scientific, Waltham, MA, USA). Afterwards, $100 \mathrm{ng}$ of an RNA sample were used for downstream analysis.

\section{Microarray expression analysis and statistics}

In the first step, the whole RNA (100 ng) from each sample was subjected to two rounds of sense cDNA amplification (Ambion ${ }^{\circledR}$ WT Expression Kit, provided by Ambion, Austin, TX, USA). The synthesis of cRNA was performed by in vitro transcription $\left(16 \mathrm{~h}, 40^{\circ} \mathrm{C}\right)$. Subsequently, cRNA was purified and retranscribed into cDNA. The cDNA samples were then used for biotin labeling and fragmentation using an Affymetrix GeneChip® WT Terminal Labeling and Hybridization kit (Affymetrix). These biotin-la- 
beled samples were loaded onto and hybridized to the Affymetrix ${ }^{\circledR}$ Porcine Gene 1.1 ST Array Strip $\left(48^{\circ} \mathrm{C} / 20 \mathrm{~h}\right)$. Hybridization was conducted at $48^{\circ} \mathrm{C}$ for $20 \mathrm{~h}$, employing an AccuBlock ${ }^{\mathrm{TM}}$ Digital Dry Bath (Labnet International, Inc., Edison, NJ, USA) hybridization oven. Subsequently, according to technical protocol, microarrays were washed and stained using an Affymetrix GeneAtlas ${ }^{\mathrm{TM}}$ Fluidics Station (Affymetrix, Santa Clara, CA, USA). The strips were scanned using an Affymetrix GeneAtlas ${ }^{\text {TM }}$ Imaging Station (Affymetrix, Santa Clara, CA, USA) and the scans of the microarrays were saved on hard drives as *.CEL files for downstream data analysis.

All of the presented analyses and graphs were performed using Bioconductor and R programming languages. Each *.CEL file was merged with a description file. The Robust Multiarray Averaging (RMA) algorithm was used in order to correct the background, and normalize and summarize the results. The statistical significance of the analyzed genes was determined by performing moderated t-statistics from the empirical Bayes method. The obtained p-value was corrected for multiple comparisons using Benjamini and Hochberg's false dis- covery rate. The selection of significantly altered genes was based on a p-value beneath 0.05 and expression higher than two-fold.

Differentially expressed genes were subjected to selection by examination of genes involved in cell migration regulation. The differentially expressed gene list (separated for up- and down-regulated genes) was uploaded to the DAVID software (Database for Annotation, Visualization and Integrated Discovery) [9], where genes belonging to the terms of all three Gene Ontology (GO) domains were extracted. Expression data of these genes were also subjected to a hierarchical clusterization procedure, and their expression values were presented as a heat map.

Subsequently, we analyzed the relation between the genes belonging to chosen GO terms with the GOplot package [10]. The GoPlot package had calculated the z-score, hence the number of up-regulated genes minus the number of down-regulated genes divided by the square root of the count. This information allowed the estimation of the change course of each gene-ontology term.

The interactions between differentially expressed genes/proteins belonging to the studied gene ontol-
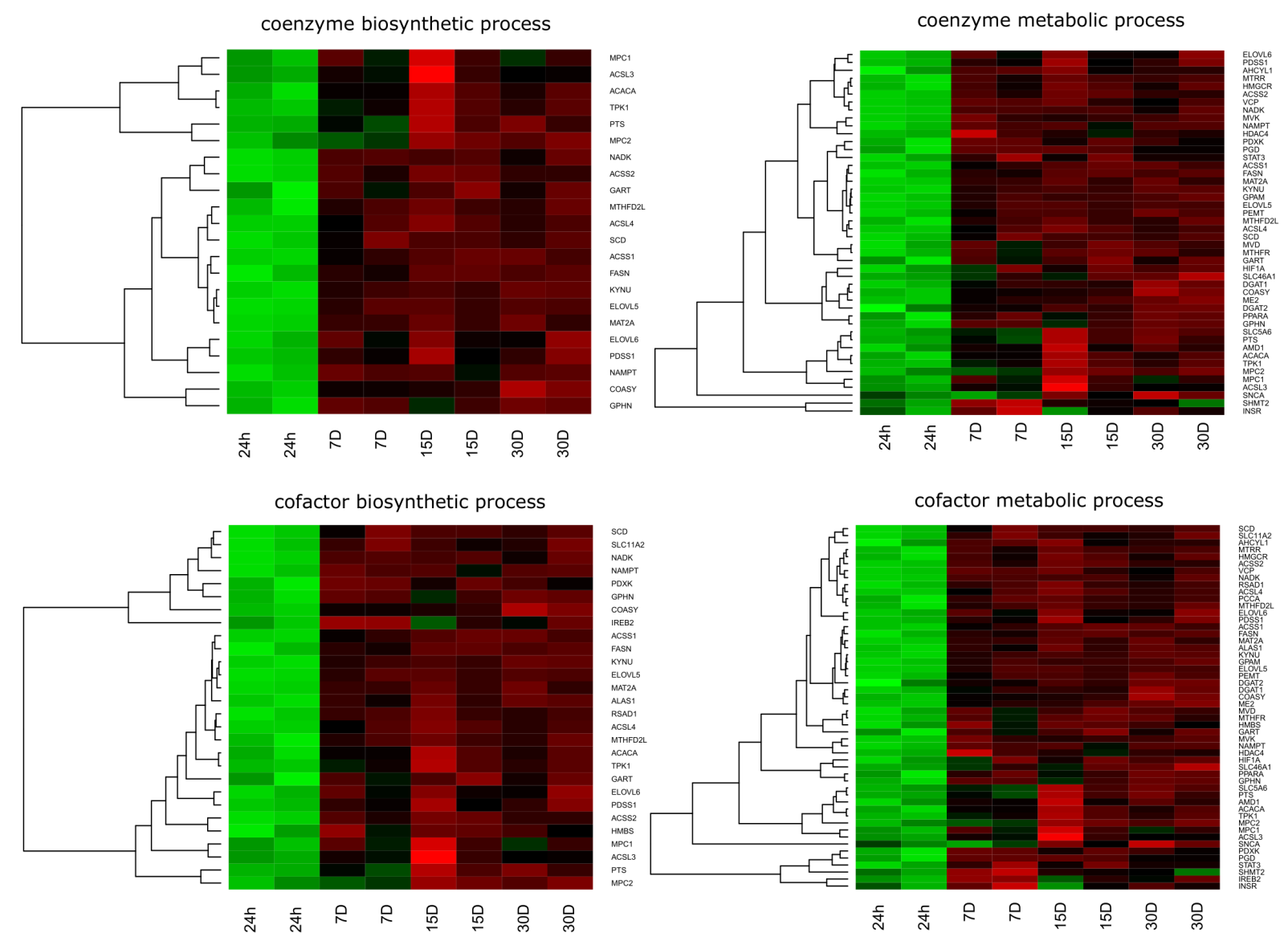

FIGURE 1 Heat map representation of differentially expressed genes belonging to the "coenzyme biosynthetic process", "coenzyme metabolic process", "cofactor biosynthetic process" and "cofactor metabolic process" GO BP terms. Arbitrary signal intensity acquired from microarray analysis is represented by colours (green higher expression; red lower expression). Log2 signal intensity values for any single gene were resized to Row Z-Score scale (from -2, the lowest expression to +2 , the highest expression for single gene) 
TABLE 1 Gene symbols, fold change in expression ratio, Entrez gene IDs, corrected p values and mean value of fold change ratio of the 10 chosen differentially expressed, studied genes

\begin{tabular}{l|c|c|c|c|c|c|c|c}
$\begin{array}{c}\text { GENE } \\
\text { SYMB0L }\end{array}$ & $\begin{array}{c}\text { RATI0 } \\
\text { D7/D1 }\end{array}$ & $\begin{array}{c}\text { RATI0 } \\
\text { D15/D1 }\end{array}$ & $\begin{array}{c}\text { RATI0 } \\
\text { D30/D1 }\end{array}$ & $\begin{array}{c}\text { ADJUSTED } \\
\text { P.VALUE } \\
\text { D7/D1 }\end{array}$ & $\begin{array}{c}\text { ADJUSTED } \\
\text { P.VALUE } \\
\text { D15/D1 }\end{array}$ & $\begin{array}{c}\text { ADJUSTED } \\
\text { P.VALUE } \\
\text { D30/D1 }\end{array}$ & $\begin{array}{c}\text { ENTREZ } \\
\text { GENE } \\
\text { ID }\end{array}$ & $\begin{array}{c}\text { MEAN } \\
\text { RATI0 }\end{array}$ \\
\hline ELOVL5 & $-9,251267471$ & $-9,938010711$ & $-10,38803385$ & 0,772063445 & 0,001410011 & 0,001221118 & 60481 & $-9,85910401$ \\
\hline GPAM & $-8,02377638$ & $-8,20961524$ & $-10,82671442$ & 0,076845334 & 0,001194058 & 0,001135003 & 57678 & $-9,020035348$ \\
\hline KYNU & $-7,126693786$ & $-8,012261249$ & $-10,12683753$ & 0,046856913 & 0,000599885 & 0,000492234 & 8942 & $-8,421930854$ \\
\hline SCD & $-7,317544673$ & $-7,853658138$ & $-7,57763343$ & 0,897789877 & 0,01064549 & 0,008292358 & 6319 & $-7,582945414$ \\
\hline MTHFD2L & $-6,467890961$ & $-8,066798079$ & $-7,468977384$ & 0,726193645 & 0,005971885 & 0,003885481 & 441024 & $-7,334555475$ \\
\hline NAMPT & $-7,606632986$ & $-5,292293151$ & $-7,422938244$ & 0,155430935 & 0,004519068 & 0,007571066 & 10135 & $-6,773954794$ \\
\hline HMGCR & $-5,271754338$ & $-7,924819598$ & $-5,887363184$ & 0,221016313 & 0,009871755 & 0,004441924 & 3156 & $-6,361312373$ \\
\hline ELOVL6 & $-4,519000381$ & $-5,545912723$ & $-5,546499245$ & 0,999794541 & 0,077215834 & 0,04794247 & 79071 & $-5,203804116$ \\
\hline TPK1 & $-2,907857277$ & $-6,352044342$ & $-4,361821755$ & 0,104734337 & 0,027816555 & 0,004611004 & 27010 & $-4,540574458$ \\
\hline ME2 & $-3,286963496$ & $-3,973472161$ & $-5,442013188$ & 0,019676685 & 0,002620929 & 0,001569971 & 4200 & $-4,234149615$ \\
\hline
\end{tabular}

D7/D1

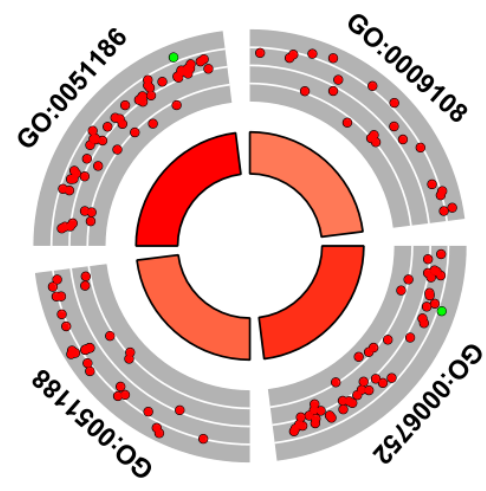

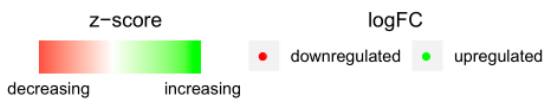

D15/D1

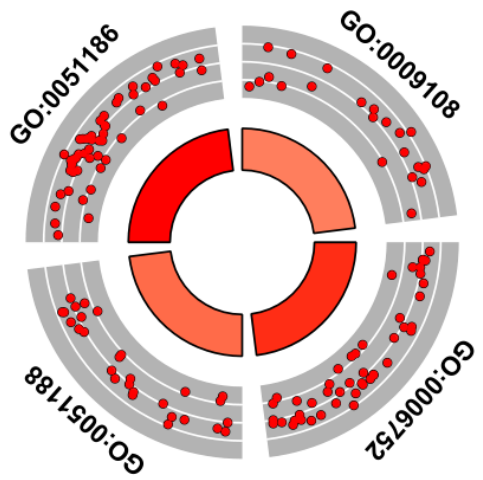

\begin{tabular}{|c|c|}
\hline ID & Description \\
\hline GO:0009108 & coenzyme biosynthetic process \\
\hline GO:0006752 & coenzyme metabolic process \\
\hline GO:0051188 & cofactor biosynthetic process \\
\hline GO:0051186 & cofactor metabolic process \\
\hline
\end{tabular}

FIGURE 2 Circular visualization of the results of gene-annotation enrichment analysis. The outer circle shows a scatter plot for each term of the logFC of the assigned genes. Green dots display up- regulation and red ones down- regulation. The inner circle is the representation of z-score. The width of the bar represents the number of genes that are present in the GO term and the color represents the z-score

ogy group were investigated by STRING10 software (Search Tool for the Retrieval of Interacting Genes) [11]. The list of gene names was used as a query for an interaction prediction. The search criteria were based on co-occurrences of genes/proteins, their co-expression, and their experimentally observed interactions in scientific texts (text mining). The results of such analyses generated a gene/protein interaction network in which the intensity of the edges reflected the strength of the interaction score.

Finally, the functional interaction between genes that belong to the chosen GO BP terms were investigated by REACTOME FIViz application to the Cy- toscape 3.6.0 software. The ReactomeFIViz app is designed to find pathways and network patterns related to cancer and other types of diseases. This app accesses the pathways stored in the Reactome database, allowing to do pathway enrichment analysis for a set of genes, visualize hit pathways using manually laid-out pathway diagrams directly in Cytoscape, and investigate functional relationships among genes in hit pathways. The app can also access the Reactome Functional Interaction (FI) network, a highly reliable and manually curated pathway-based protein functional interaction network covering over $60 \%$ of human proteins. 


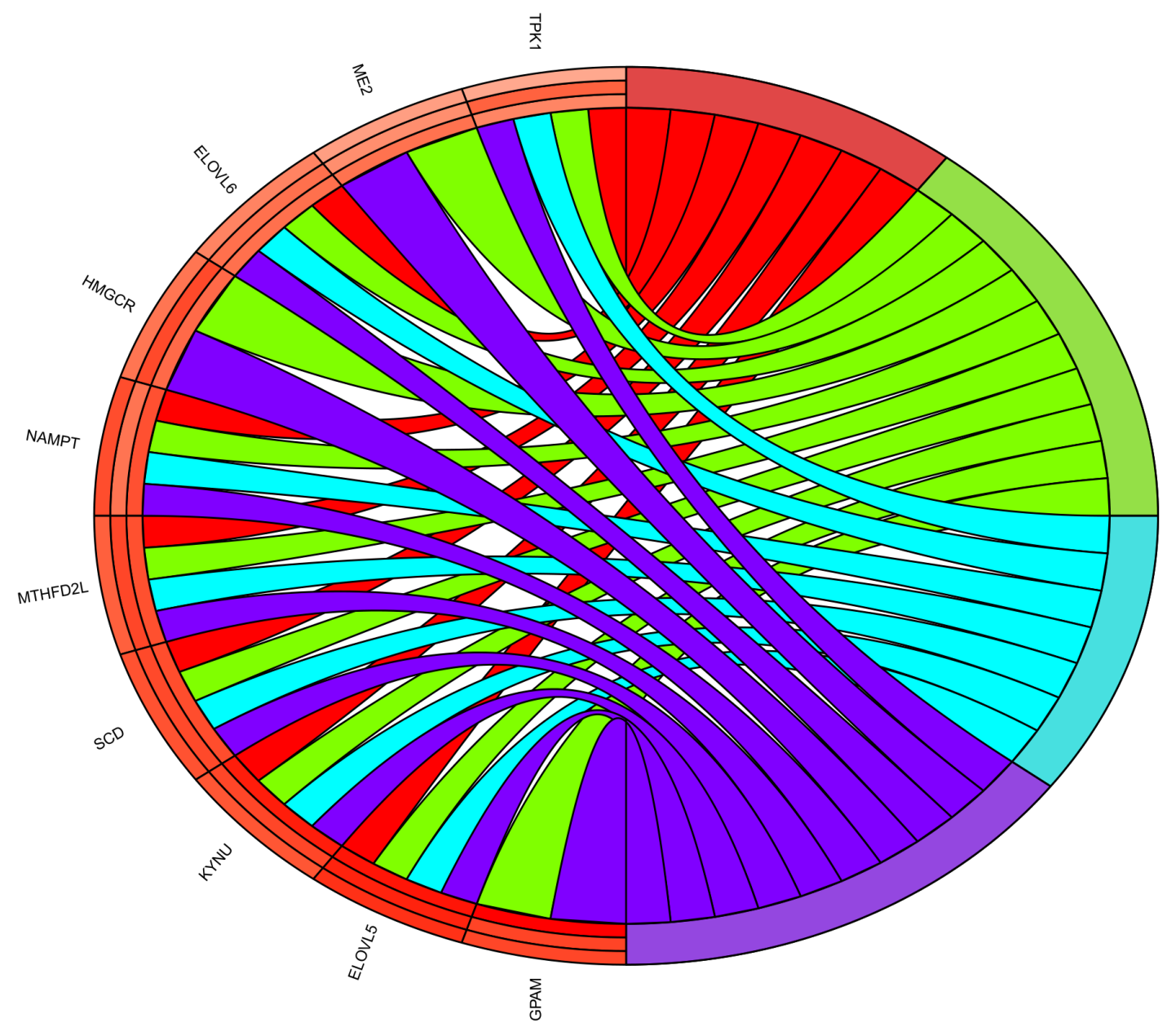

GO Terms

coenzyme biosynthetic process

coenzyme metabolic process

cofactor biosynthetic process

cofactor metabolic process

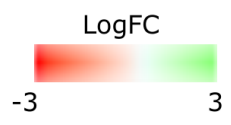

FIGURE 3 Representation of the mutual relationship between differently expressed genes that belong to 10 chosen genes from "coenzyme biosynthetic process", "coenzyme metabolic process", "cofactor biosynthetic process" and "cofactor metabolic process" GO BP terms. The ribbons indicate which gene belongs to which category. The middle circle shows the logarithm of the fold change (LogFC) between D7/D1, D15/D1 and D30/D1 respectively. The color of each block corresponds to the LogFC of each gene (green - upregulated, red - downregulated). The genes were sorted by LogFC from most to least changed gene

\section{Ethical approval}

The research has been complied with all the relevant national regulations, institutional policies and in accordance the tenets of the Helsinki Declaration, and has been approved by the authors' institutional review board or equivalent committee. Poznan University of Medical Sciences Bioethics Committee approval no. 32/2012 from June 1, 2012.

\section{Results}

Whole transcriptome profiling by Affymetrix microarray allows to analyze the gene expression changes of the oocyte after in vitro maturation in relation to freshly isolated oocyte before in vitro procedure (in Vivo). By Affymetrix ${ }^{\circledR}$ Porcine Gene 1.1 ST Array the expression of 22480 transcripts were examined. Genes were considered as differentially expressed when presented fold changes higher than abs (2) and corrected p value lower than 0.05 . This set of genes consist of 2278 different transcripts. The first detailed analysis based on GO BP lead to the identification of differentially expressed genes belonging to the significantly enrichment GO BP terms.

The DAVID (Database for Annotation, Visualization and Integrated Discovery) software was used for the extraction of the genes belonging to the gene ontology biological process terms (GO BP). Up and down regulated gene sets were subjected to DAVID searching separately and only gene sets where adj. $\mathrm{p}$ values were lower than 0.05 were selected. The DAVID software analysis showed that differently expressed genes belong to 582 Gene ontology groups and 45 KEGG pathways. The genes of main focus were "coenzyme biosynthetic process", "coenzyme metabolic process", "cofactor biosynthetic process" 


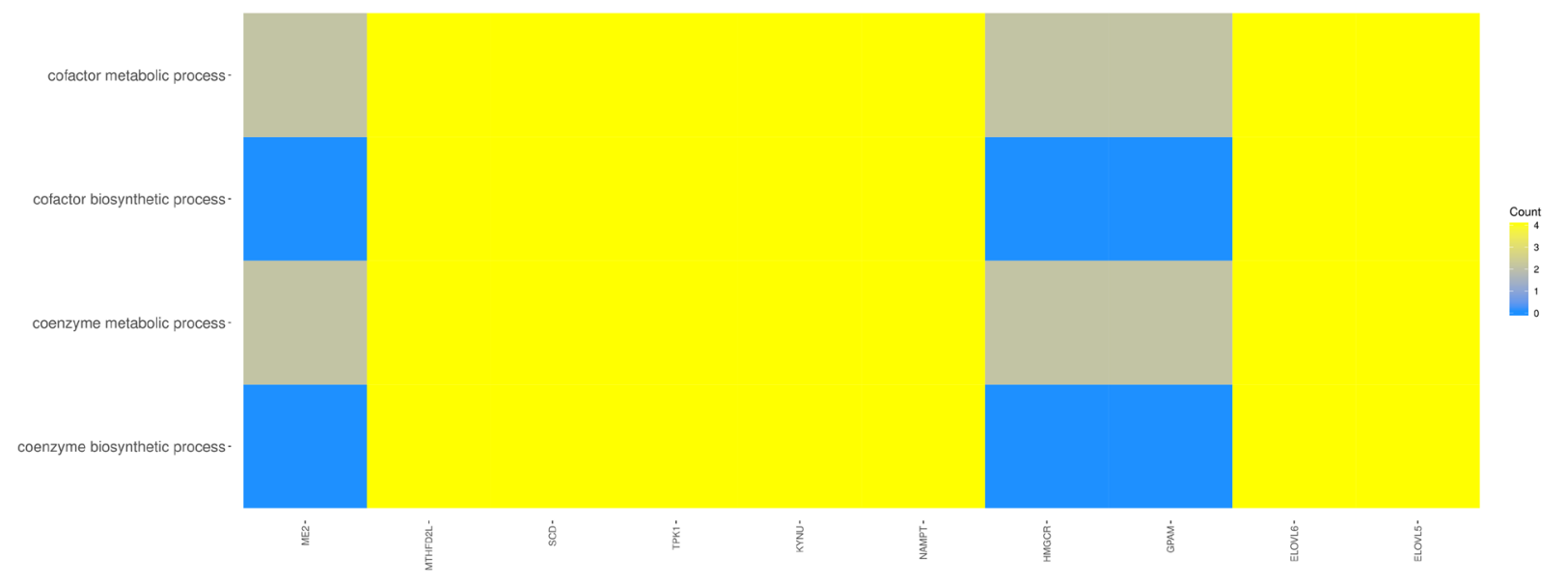

FIGURE 4 Heatmap showing the gene occurrence between the chosen 10 differently expressed genes that belong to "coenzyme biosynthetic process", "coenzyme metabolic process", "cofactor biosynthetic process" and "cofactor metabolic process" GO BP terms. The yellow color is associated with gene occurrence in the GO Term. The intensity of the color corresponds to the amount of GO BP terms that each gene belongs to

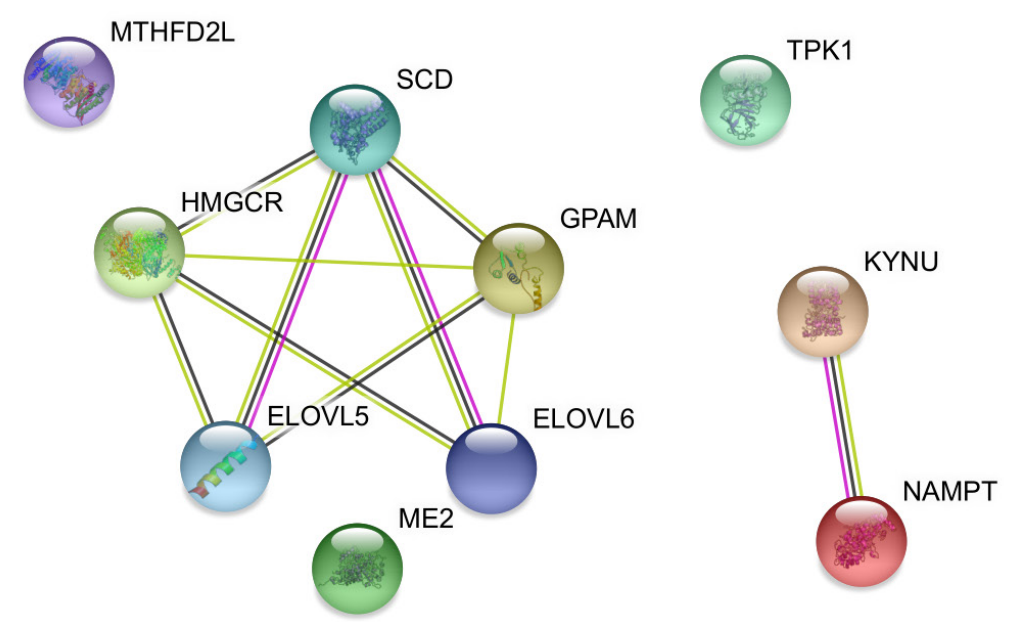

Known interaction

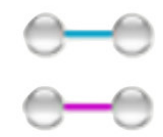

Predicted interactions

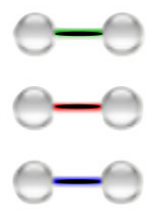

gene neighborhood

gene fusions

gene co-occurrence
Others

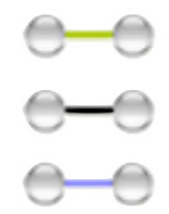

textmining

co-expression

protein homology

FIGURE 5 STRING-generated interaction network among 10 most downregulated genes belonging to the "coenzyme biosynthetic process", "coenzyme metabolic process", "cofactor biosynthetic process" and "cofactor metabolic process" GO BP terms. The intensity of the edges reflects the strength of the interaction scores

and "cofactor metabolic process" GO BP terms. These sets of genes were subjected to hierarchical clusterization procedure and presented as heatmaps (Fig. 1). The symbols of genes, fold changes in expression, Entrez gene IDs and corrected p values of that genes are shown in table $\mathbf{1}$. The enrichments of each GO BP term were calculated as z-score and they are shown on a circle diagram (Fig. 2)

In the Gene Ontology database, genes that formed one particular GO group can also belong to other different GO term categories. By this meaning, the gene intersections between selected GO BP terms were explored and the relation between the GO BP terms are presented as circle plot (Fig. 3) as well as heatmap (Fig. 4).

The STRING software generated interaction networks among differentially expressed genes belonging to each of the selected GO BP terms. Through this method it was possible to observe the molecular interaction network formed between protein products of studied genes (Fig. 5). Finally, the functional interactions between chosen genes were investigated with REACTOME FIViz app to Cytoscape 3.6.0 software and the results are shown in figure 6 . 

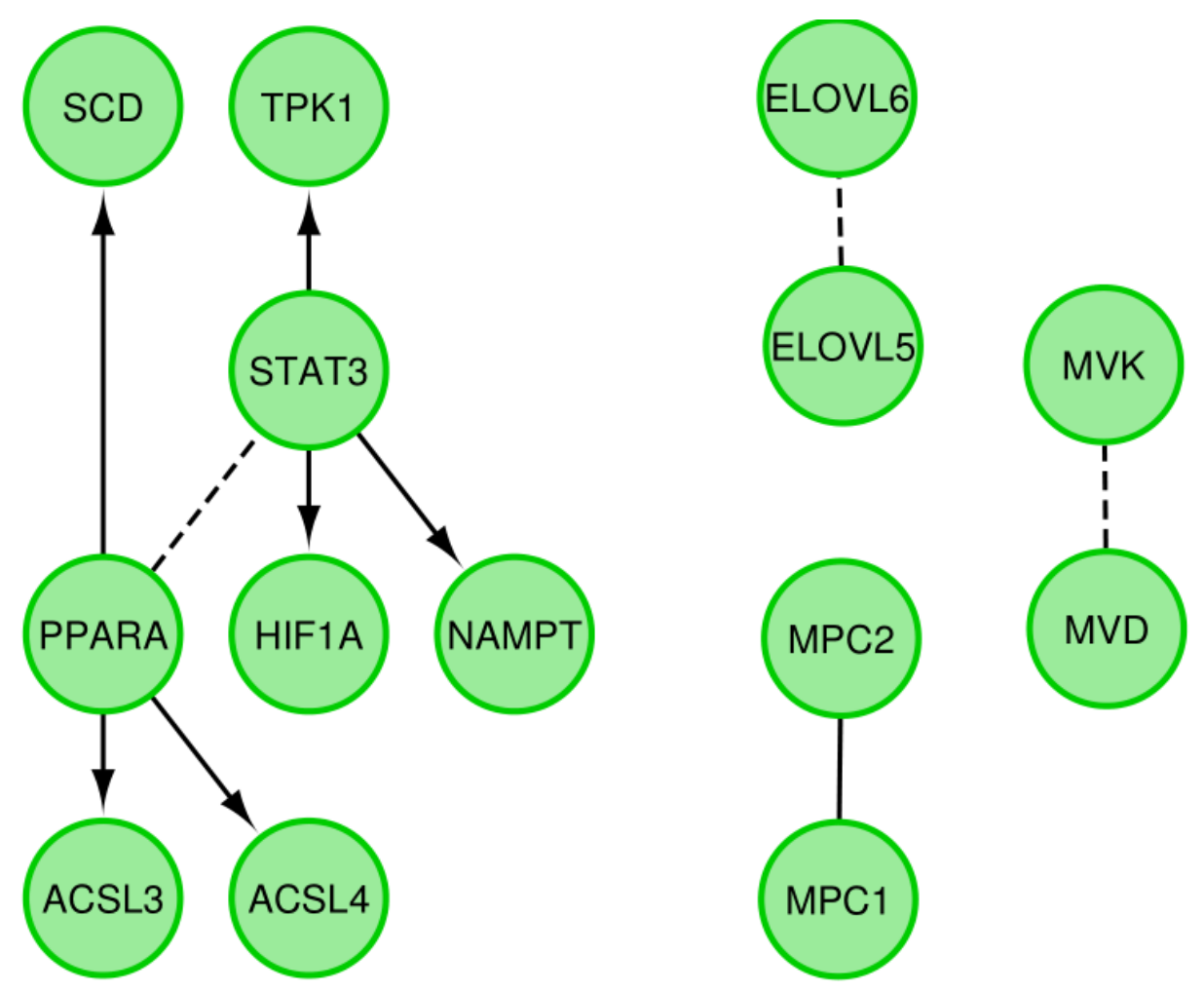

FIGURE 6 Functional interaction (FI) between differently expressed genes that belong to the "coenzyme biosynthetic process", "coenzyme metabolic process", "cofactor biosynthetic process" and "cofactor metabolic process" GO BP terms. In the following figure "->" stands for activating/catalyzing, "-|" for inhibiting, "-" for FIs extracted from complexes or inputs, and "---" for predicted FIs

\section{Discussion}

GCs are known to be closely related to mammalian oocytes. GCs surround the oocyte and they form the proper architecture of the ovarian follicle. In physiological conditions, GCs support the development and the maturation of the oocyte, and the formation of ovarian follicles [12]. Moreover, they produce gap junctions that allow a bidirectional communication with the oocyte. Thus, in addition to the hormonal secretory function, GCs also play an important role in communication with oocytes. They are associated with the functioning of the reproductive system, as well as the maintenance of pregnancy through the participation in the synthesis of steroid hormones. After ovulation, when the follicle ruptures, the GCs fill the formed gap and transform into lutein cells, whose main purpose is to produce progesterone and maintain pregnancy. The resulting structure is called the corpus luteum [13]. Understanding the molecular mechanisms regulating this process is pivotal to completely understand the regulation of oogenesis and the ovarian function.

The physiological and morphological changes that occur in GCs during the formation of the ovarian follicle have been well characterized. However, the molecular mechanisms and gene interactions of GCs are still insufficiently described, Previous studies are an important introduction to a better understanding of all key mechanisms in this cell population [13]. In addition to indicating numerous molecular markers that are key to many physiological and biochemical processes occurring in these cells [13-15], the very high plasticity of GCs and their potential to differentiate into other cell populations were demonstrated[16-18]. In the present study, by employing primary in vitro long-term culture and microarray approach, groups of genes associated with enzymatic reactions were described.

Of all the genes showed on the heat maps in Fig. 1 and belonging to GO BP terms of interest, the greatest reduction in the level of mRNA expression has been observed in the case of the gene ELOVL5. To date, seven enzymes have been identified, termed ELOVL 1-7 (Elongation of very-long-chain fatty acid) they are suggested to perform the condensation reaction in the elongation cycle, [19]. Longchain fatty acid elongase 5 (Elovl5) is the rate-controlling enzyme that catalyzes the elongation reaction of long-chain polyunsaturated fatty acids (PUFAs), including arachidonic acid, oleic acid, stearidonic acid, palmitic acid, linolenic acid and eicosapentaenoic acid. These fatty acids (FA) not only are an essential components of the cell membrane, but they also participate in many important signaling processes [20,21]. Accordingly, their proper elongation and desaturation of are essential to the maintenance of lipid homeostasis and the disruption of these processes may have devastating consequences. Studies using ovaries of slaughterhouse origin have revealed correlations between FA content in 
follicular fluid (FF) and the expression levels of several genes, including ELOVL5, in GCs[22]. These results confirmed the importance of lipid metabolism balance in the interaction between oocytes and maternal environment. A study by Warzych et al. confirmed the distinct roles of cumulus cells (CCs) and GCs in energy metabolism of the follicle. In fact, they showed different ELOVL5 transcript levels (GCs were characterized by significantly higher mRNA levels), regardless of the donor [23]. Almiñana et al., demonstrated presence of ELOVL5 transcripts in oviductal extracellular vesicles (OEVs), which contain oviductal secretions [24].

The detection of the reduced mRNA expression level of another ELOVL family member, ELOVL6 is an additional argument suggesting decreased activity of elongation very long chain fatty acids (VLCFA) in GCc during in vitro conditions. ELOVL6 protein, in contrast to ELOVL5, prefers saturated and mono-unsaturated fatty acids as substrates in the elongation pathway [25]. A studies provided by Annes et al. compared the expression of lipid-related genes and follicular fluid molecules between different bovine follicle sizes. It was demonstrated that ELOVL6 showed lower transcript levels in larger follicle-derived ( $\geq 6 \mathrm{~mm}$ ) oocytes compared with the $\leq 2 \mathrm{~mm}$ group [26]. Another study provided a comprehensive description of stage-specific lipidome signatures in early bovine embryo development, via assessment the mRNA abundance of lipid metabolism-related genes (including both ELOVL5 and ELOVL6). When compared to earlier stages (immature oocytes, two-cell embryos, and eight- to 16cell embryos), ELOVL5 and ELOVL6 mRNA quantity increased significantly at morula stage, followed by a decrease at blastocyst stage. The authors proved that both ELOVL family members overexpression preceded an increased abundance of a series of lipid species at blastocyst stage [27].

Significantly reduced GPAM transcript level also confirms the importance of changes at the molecular level in the enzymatic regulation of fatty acid metabolism. GPAM (Glycerol-3-phosphate acyltransferase mitochondrial) is a gene that encodes a mitochondrial enzyme which catalyzes the initial and committed step of glycerolipids synthesis and that prefers saturated fatty acids as its substrates for the catalyzed biosynthesis process [28]. Transcriptomic studies on molecular regulation of FA metabolism in 3-8 mm ovarian follicles of bovine have shown high GPAM transcript expression in theca (TH), GCs, and also in CCs [29]. Subsequent studies of Yu laboratory [30] showed that GPAM gene can promote the synthesis of triglycerides in the fat metabolism pathway of bovine embryonic fibroblast cells. Especially important conclusions provided by Marchan et al. indicated that analysis of GPAM expression in different cancer types revealed a significant association between high GPAM expression and reduced overall survival in ovarian cancer. The authors' results clearly showed how GPAM levels influence intracellular lysophosphatidic acid (LPA) levels to promote cell migration and tumor growth [31].

\section{Conclusions}

Decreasing levels of gene expression involved in the functioning of the mechanism of enzymatic reactions occur over the course of long-term cell culture. The transcriptomic profile variability for genes (ELOVL5, ELOVL6 and GPAM) involved in enzymatic regulation of fatty acid metabolism illustrated a down regulation in these genes. It is likely that the reduced enzymatic activity over culture indicates lower expression levels compared to in vivo situation.

\section{Corresponding author}

Corresponding author: Bartosz Kempisty PhD, Department of Histology and Embryology, Department of Anatomy, Poznan University of Medical Sciences, 6 Święcickiego St., 60-781 Poznań, Poland Tel./Fax: +48 618546418 / +48 61 8546440, e-mail: bkempisty@ump.edu.pl.

\section{Conflict of interest statement}

The authors declare they have no conflict of interest.

\section{References}

1. Lan CW, Chen MJ, Tai KY, Yu DC, Yang YC, Jan PS, Yang YS, Chen HF, Ho HN. Functional microarray analysis of differentially expressed genes in granulosa cells from women with polycystic ovary syndrome related to MAPK/ERK signaling. Sci Rep. 2015;5; DOI:10.1038/srep14994.

2. Orisaka M, Tajima K, Tsang BK, Kotsuji F. Oocyte-granulosa-theca cell interactions during preantral follicular development. J Ovarian Res. 2009;2; DOI:10.1186/1757-2215-2-9.

3. Uyar A, Torrealday S, Seli E. Cumulus and granulosa cell markers of oocyte and embryo quality. Fertil. Steril., vol. 99, 2013, p. 979-97; DOI:10.1016/j.fertnstert.2013.01.129.

4. Buganim Y, Jaenisch R. Transdifferentiation by defined factors as a powerful research tool to address basic biological questions. Cell Cycle. 2012;11:4485-6; DOI:10.4161/cc.22665.

5. Dzafic E, Stimpfel M, Virant-Klun I. Plasticity of granulosa cells: on the crossroad of stemness and transdifferentiation potential. J Assist Reprod Genet. 2013;30:1255-61; DOI:10.1007/s10815-013-0068-0.

6. Kossowska-Tomaszczuk K, De Geyter C, De Geyter M, Martin I, Holzgreve W, Scherberich A, Zhang H. The Multipotency of Luteinizing Granulosa Cells Collected from Mature Ovarian Follicles. Stem Cells. 2009;27:2109; DOI:10.1634/stemcells.2008-0233.

7. Kossowska-Tomaszczuk K, De Geyter C. Cells with Stem Cell Characteristics in Somatic Compartments of the Ovary. Biomed Res Int. 2013;2013:1-8; DOI:10.1155/2013/310859.

8. Chomczynski P, Sacchi N. Single-step method of RNA isolation by acid guanidinium thiocyanate-phenol-chloroform extraction. Anal Biochem. 1987;162:156-9; DOI:10.1016/0003-2697(87)90021-2.

9. Huang DW, Sherman BT, Tan Q Kir J, Liu D, Bryant D, Guo Y, Stephens R, Baseler MW, Lane HC, Lempicki RA. DAVID Bioinformatics Resources: expanded annotation database and novel algorithms to better extract biology from large gene lists. Nucleic Acids Res. 2007;35:W169-75; DOI:10.1093/nar/gkm415.

10. Walter W, Sánchez-Cabo F, Ricote M. GOplot: an R package for visually combining expression data with functional analysis: Fig. 1. Bioinformatics. 2015;31:2912-4; DOI:10.1093/bioinformatics/btv300.

11. von Mering C, Jensen LJ, Snel B, Hooper SD, Krupp M, Foglierini M, Jouffre N, Huynen MA, Bork P. STRING: known and predicted protein-protein associations, integrated and transferred across organisms. Nucleic Acids Res. 2005;33:D433-7; DOI:10.1093/nar/gki005.

12. Ciesiółka S, Budna J, Jopek K, Bryja A, Kranc W, Chachuła A, Borys S, Dyszkiewicz Konwińska M, Ziółkowska A, Antosik P, Bukowska D, Brüssow KP, Bruska M, Nowicki M, Zabel M, Kempisty B. Influence of Estradiol-17beta on Progesterone and Estrogen Receptor mRNA Expression in Porcine Follicular Granulosa Cells during Short-Term, in Vitro Real-Time Cell Proliferation. Biomed Res Int. 2016;2016; DOI:10.1155/2016/8431018.

13. Kranc W, Budna J, Kahan R, Chachuła A, Bryja A, Ciesiółka S, Borys S, Antosik MP, Bukowska D, Brussow KP, Bruska M, Nowicki M, Zabel M, 
Kempisty B. Molecular basis of growth, proliferation, and differentiation of mammalian follicular granulosa cells. J Biol Regul Homeost Agents. 2017;31:1-8.

14. Kranc W, Brązert M, Ożegowska K, Nawrocki M, Budna J, Celichowski $P$ Dyszkiewicz-Konwińska M, Jankowski M, Jeseta M, Pawelczyk L, Bruska M, Nowicki M, Zabel M, Kempisty B. Expression Profile of Genes Regulating Steroid Biosynthesis and Metabolism in Human Ovarian Granulosa Cells-A Primary Culture Approach. Int J Mol Sci. 2017;18:2673; DOI:10.3390/ijms18122673.

15. Chermuła B, Brazert M, Izycki D, Ciesiółka S, Kranc W, Celichowski P, Ozegowska K, Nawrocki MJ, Jankowski M, Jeseta M, Antosik P, Bukowska D, Skowroński MT, Brussow KP, Bruska M, Pawelczyk L, Zabel M, Nowicki M, Kempisty B. New Gene Markers of Angiogenesis and Blood Vessels Development in Porcine Ovarian Granulosa Cells during Short-Term Primary Culture in Vitro. Biomed Res Int. 2019;2019; DOI:10.1155/2019/6545210.

16. Kranc W, Brązert M, Budna J, Celichowski P, Bryja A, Nawrocki MJ, Ożegowska K, Jankowski M, Chermuła B, Dyszkiewicz-Konwińska M, Jeseta M, Pawelczyk L, Bręborowicz A, Rachoń D, Bruska M, Nowicki M, Zabe M, Kempisty B. Genes responsible for proliferation, differentiation, and junction adhesion are significantly up-regulated in human ovarian granulosa cells during a long-term primary in vitro culture. Histochem Cell Biol. 2019;151:125-43; DOI:10.1007/s00418-018-1750-1.

17. Kulus M, Sujka-Kordowska P, Konwerska A, Celichowski P, Kranc W, Kulus J, Piotrowska-Kempisty H, Antosik P, Bukowska D, Iżycki D, Bruska M, Zabel M, Nowicki M, Kempisty B. New Molecular Markers Involved in Regulation of Ovarian Granulosa Cell Morphogenesis, Development and Differentiation during Short-Term Primary In Vitro Culture-Transcriptomic and Histochemical Study Based on Ovaries and Individual Separated Follicles. Int J Mol Sci. 2019;20:3966; DOI:10.3390/ijms20163966.

18. Kranc W, Brązert M, Celichowski P, Bryja A, Nawrocki MJ, Ożegowska K, Jankowski M, Jeseta M, Pawelczyk L, Bręborowicz A, Rachoń D, Skowroński MT, Bruska M, Zabel M, Nowicki M, Kempisty B. 'Heart development and morphogenesis' is a novel pathway for human ovarian granulosa cell differentiation during long-term in vitro cultivation-a microarray approach. Mol Med Rep. 2019;19:1705-15; DOI:10.3892/ mmr.2019.9837.

19. Jakobsson A, Westerberg R, Jacobsson A. Fatty acid elongases in mammals: Their regulation and roles in metabolism. Prog Lipid Res. 2006;45:237-49; DOI:10.1016/j.plipres.2006.01.004.

20. Guillou H, Zadravec D, Martin PGP, Jacobsson A. The key roles of elongases and desaturases in mammalian fatty acid metabolism: Insights from transgenic mice. Prog Lipid Res. 2010;49:186-99; DOI:10.1016/j. plipres.2009.12.002

21. Denic V, Weissman JS. A Molecular Caliper Mechanism for Determining Very Long-Chain Fatty Acid Length. Cell. 2007;130:663-77; DOI:10.1016/j.cell.2007.06.031.

22. Warzych E, Pawlak P, Pszczola M, Cieslak A, Madeja ZE, Lechniak D. Interactions of bovine oocytes with follicular elements with respect to lipid metabolism. Anim Sci J. 2017;88:1491-7; DOI:10.1111/asj.12799.

23. Warzych E, Pawlak P, Pszczola M, Cieslak A, Lechniak D. Prepubertal heifers versus cows-The differences in the follicular environment. Theriogenology. 2017;87:36-47; DOI:10.1016/j. theriogenology.2016.08.007.

24. Almiñana C, Tsikis G, Labas V, Uzbekov R, da Silveira JC, Bauersachs S Mermillod P. Deciphering the oviductal extracellular vesicles content across the estrous cycle: Implications for the gametes-oviduct interactions and the environment of the potential embryo. BMC Genomics. 2018;19; DOI:10.1186/s12864-018-4982-5.

25. Wang Y, Botolin D, Christian B, Busik J, Xu J, Jump DB. Tissue-specific, nutritional, and developmental regulation of rat fatty acid elongases. J Lipid Res. 2005;46:706-15; DOI:10.1194/jlr.M400335-JLR200.

26. Annes K, Müller DB, Vilela JAP, Valente RS, Caetano DP, Cibin FWS, Milazzotto MP, Mesquita FS, Belaz KRA, Eberlin MN, Sudano MJ. Influence of follicle size on bovine oocyte lipid composition, follicular metabolic and stress markers, embryo development and blastocyst lipid content. Reprod Fertil Dev. 2019;31:462-72; DOI:10.1071/RD18109.

27. Sudano MJ, Rascado TDS, Tata A, Belaz KRA, Santos VG, Valente RS, Mesquita FS, Ferreira CR, Araújo JP, Eberlin MN, Landim-Alvarenga FDC. Lipidome signatures in early bovine embryo development. Theriogenology. 2016;86:472-484.e1; DOI:10.1016/j.theriogenology.2016.03.025.

28. Brockmöller SF, Bucher E, Müller BM, Budczies J, Hilvo M, Griffin JL, Orešič M, Kallioniemi O, Iljin K, Loibl S, Darb-Esfahani S, Sinn B V., Klauschen F, Prinzler J, Bangemann N, Ismaeel F, Fiehn O, Dietel M, Denkert C. Integration of metabolomics and expression of glycerol-3-phosphate acyltransferase (GPAM) in breast cancer-link to patient survival, hormone receptor status, and metabolic profiling. J Proteome Res. 2012;11:850-60; DOI:10.1021/pr200685r.
29. Bertevello PS, Teixeira-Gomes AP, Seyer A, Carvalho AV, Labas V, Blache MC, Banliat C, Cordeiro LAV, Duranthon V, Papillier P, Maillard V, Elis S, Uzbekova S. Lipid identification and transcriptional analysis of controlling enzymes in bovine ovarian follicle. Int J Mol Sci. 2018;19; DOI:10.3390/ijms19103261.

30. Yu H, Zhao Z, Yu X, Li J, Lu C, Yang R. Bovine lipid metabolism related gene GPAM: Molecular characterization, function identification, and association analysis with fat deposition traits. Gene. 2017;609:9-18; DOI:10.1016/j.gene.2017.01.031.

31. Marchan R, Büttner B, Lambert J, Edlund K, Glaeser I, Blaszkewicz M, Leonhardt G, Marienhoff L, Kaszta D, Anft M, Watzl C, Madjar K, Grinberg M, Rempel E, Hergenröder R, Selinski S, Rahnenführer J, Lesjak MS, Stewart JD, Cadenas C, Hengstler JG. Glycerol-3-phosphate acyltransferase 1 promotes tumor cell migration and poor survival in ovarian carcinoma. Cancer Res. 2017;77:4589-601; DOI:10.1158/0008-5472. CAN-16-2065. 